NASA

Technical Memorandum 106674
Army Research Laboratory Technical Report ARL-TR-572

$p=16$

\title{
An Aerodynamic Analysis of a Mixed Flow Turbine
}

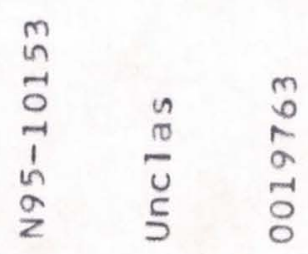

Chan M. Kim

National Aeronautics and Space Administration Lewis Research Center

Cleveland, Ohio

and

Kestutis C. Civinskas

Vehicle Propulsion Directorate

U.S. Army Research Laboratory

Lewis Research Center

Cleveland, Ohio

Prepared for the

30th Joint Propulsion Conference

cosponsored by AIAA, ASME, SAE, and ASEE

Indianapolis, Indiana, June 27-29, 1994

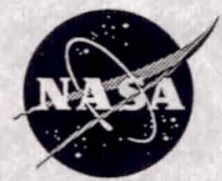

National Aeronautics and

Space Administration

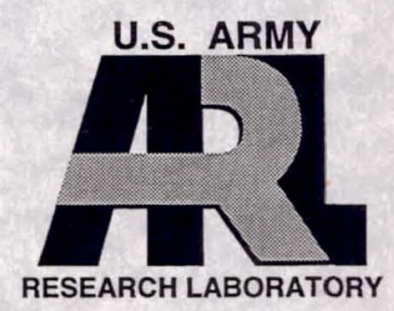


An Aerodynamic Analysis of a Mixed Flow Turbine

\author{
Chan M. Kim \\ NASA Lewis Research center \\ Cleveland, Ohio 44135 \\ and \\ Kestutis C. Civinskas \\ Vehicle Propulsion Directorate (ARL) \\ Cleveland, Ohio 44135
}

\section{Abstract}

The aerodynamic performance of a high-work mixed flow turbine (MFT) is computed and compared with experimental data. A three dimensional (3-D) viscous analysis is applied to the single stage MFT geometry with a relatively long upstream transition duct. Predicted vane surface static pressures and circumferentially averaged spanwise quantities at stator and rotor exits agree favorably with data. Compared to the results of axisymmetric flow analysis from design intent, the 3-D computation agrees much better especially in the endwall regions where throughflow prediction fails to assess the loss mechanism properly. Potential sources of performance loss such as tip leakage and secondary flows are also properly captured by the analysis.

\section{Introduction}

Enhancing turbine inlet temperature to improve the cycle performance is a driving factor in the development of advanced turbine materials. For small gas turbine engines, a ceramic radial turbine can offer a competitive edge over an axial machine. However, the high blade tip speed and higher risk of particle impact damage on the blades are major structural constraints for ceramic radial turbines. Although swept blading or a high-work axial turbine may be considered alternate design options (Rodgers [1]), a mixed flow turbine (MFT) can provide some advantages through its reduced tip speed and oblique blade angles without compromising the performance. okapuu [2], through rotating rig testing, demonstrated the efficiency benefit of MFT over that of axial and radial turbines for moderate pressure ratio and work output application.

A very limited number of analytical studies on the MFT has been reported in the open literature. Civinskas [3] investigated the aerodynamic performance of four MFT configurations using a quasi-3D flow and boundary layer analysis approach of Boyle et al. [4] and showed the aerodynamic feasibility of a backswept MFT. Okapuu applied 3-D Euler analysis for the MFT in reference 2 , but the 
comparison with data was limited to the static pressure distribution at the nozzle midspan. Kirtley et al. [5] applied Adamczyk's 3-D average passage code [6] to analyze a transonic, single stage MFT. Although the circumferentially averaged spanwise quantities at the vane and blade exits were in good agreement with design intent, a comparison with experimental data was not available.

In the present work, the aerodynamic performance of the high-work, single stage MFT is analyzed and compared with experimental data using a three-dimensional Navier-stokes code referred to as ADPAC (Advanced Ducted Propfan Analysis Codes). Spanwise flow quantities at the stator and the rotor exits as well as the vane surface static pressures are compared with data. Computed overall performance is also compared with measurement and potential sources of performance losses are identified.

\section{Method of Analysis}

ADPAC is a threedimensional, time-dependent Euler/Navier-stokes code and is based on an explicit RungeKutta time-marching algorithm employing a finite volume, multiple blocked grid discretization. The code is constructed such that the algorithm may be applied to multiple blocked grid mesh systems with common grid interface boundaries. Several convergence enhancements are added for the prediction of steady state flows including local time stepping, implicit residual smoothing, and multigrid. The Baldwin-Lomax turbulence model [7] is adopted to simulate the effects of turbulence. The code was developed by Hall and Delaney and details can be found in [8].

For the analysis of more than a single isolated blade row, the code has features of either solving the timeresolved unsteady equations or performing steady computations based on the mixing plane concept. Considering the computational expense of the unsteady option, the present study adopts the steady approach. A mixing plane is an arbitrarily imposed boundary inserted between adjacent blade rows across which the flow is mixed out circumferentially. This circumferential mixing approximates the time-averaged condition at the mixing plane and allows the aerodynamic solution for each blade passage to be performed in a steady flow environment. In this approach, flow variables on either side of the mixing plane are circumferentially averaged and passed to the neighboring blade row as a means of smearing out the circumferential nonuniformities resulting from dissimilar blade counts.

The radial distributions of total pressure, total temperature, radial flow angle, and circumferential flow angle are specified at the grid inlet. The upstream running Riemann invariant is then extrapolated to the inlet, and along with the equation of state and specified boundary 
conditions, all other inflow variables are determined. With a relatively long transition duct upstream of the stator in the present study, constant values of total pressure, total temperature, and flow angles are specified at the inlet. For the outflow boundary, the static pressure at the hub is specified and the remaining pressures are calculated by integrating the radial momentum equation. All other outflow variables are extrapolated from within. No-slip boundary conditions are employed at all solid surfaces and adiabatic walls are specified. In the tip clearance regions, periodicity is imposed across the thickness of the blade. As mentioned earlier, the mixing plane concept is utilized at the interface of stator and rotor geometry such that the flow variables are each circumferentially averaged and passed between blocks.

An H-type computational grid was generated using the TCGRID code of chima [9]. Depicted in Figure 1 (a) is the axisymmetric view of the grid geometry. The grid is made up of two blocks with the block boundary located between the stator and the rotor. The first block corresponds to the stator section preceded by a long upstream transition duct and has a grid size of 161 (streamwise), 37 (blade to blade), and 33 (spanwise). The rotor grid has a size of 121 , 37 , and 33. The computational rotor tip clearances were set at 0.016 and 0.023 inches at the leading and the trailing edges respectively. Although these clearances are not the same as measured axial and radial clearances of 0.035 and 0.022 inches of the test rig, matching the tip clearance at the trailing edge should closely simulate the actual performance. For later reference, the test locations of stator and rotor exit survey are also indicated. Note that the stator exit survey was performed at the plane of the rotor leading edge. The rotor exit survey plane is 0.33 inches from the trailing edge. Figure 1(b) shows locations of the leading and trailing edges for the stator and the rotor.

The solution procedure using ADPAC fully utilizes the multigrid scheme to accelerate convergence by initializing the solution on a coarse grid before incurring the expense of fine grid iterations. Three levels of multigrid were employed for the present study. steady state solutions were deemed converged when the average residual was reduced by

a factor of $10^{-3}$.

\section{MFT Design and Performance Test}

The MFT hardware is a single stage, high-work turbine that has been designed and tested by Allied Signal Auxiliary Power Division under a NASA/U.S. Army contract. Details of the MFT stator and rotor design and the performance test are reported in [10] and only a brief description is given here. The stator has 19 vanes with a trailing edge thickness of 0.03 inch and 
aspect ratio of 0.256 . Average vane height is 0.194 inch and meridional chord length is 0.759 inch. The vane sections are stacked at the trailing edge with a non-linear stagger angle distribution between hub and shroud. The rotor has 15 blades with cone angle of 68 degrees, 1.9 inch axial length, and an elliptic leading edge of 2:1 aspect ratio. The design axial and radial clearances are 0.02 inches. The MFT geometry which was generated using computer graphics is shown in Figure 2.

The experiment was performed in two separate tests. Test 1 , stage performance test, measured the performance of the entire stage and Test 2 was the stator exit survey test. The turbine rig for Test 2 is identical to that of Test 1 except the rotor used in Test 2 had its leading edge cut back by 0.13 inch to allow the probe to survey in the plane of the original blade leading edge. The design point test conditions in Table 1 show this slight difference. Here the design point for Test 2 was defined so that the conditions at the exit of the stator were the same as in Test 1 . This was achieved by adjusting the stator inlet total temperature and the total-to-static pressure ratio, corrected speed, and corrected flow to Test 1 values.

\section{Results and Discussion}

The computational results are compared with the test data as well as with the design intent. The design intent was conceived using an axisymmetric analysis code which includes radial turbine correlations. Table 2 compares the mass flow rate and stage total-to-total efficiency for each of these conditions at $100 \%$ speed. ADPAC results are computed from mass-averaged values of mass flow, total pressure, and total temperature at the exit. The design intent shows a substantial deviation from measured efficiency. It is speculated that the design intent which is based on the radial turbine correlations does not properly assess the performance losses for the MFT geometry considered which is three dimensional in vane geometry. The mass flow rate and efficiency from the ADPAC computation also show slightly higher values than measured ones. Part of this discrepancy may be due to the measured axial clearance of 0.035 inches of the test rig which is different from that used in design intent prediction(0.02 inches) and ADPAC calculation(0.016 inches). other possibilities of the discrepancy include the effect of rotor-stator interaction and limitations of the turbulence model.

Depicted in Figure 3 is
the vane surface static pressure distribution at the hub and shroud. The pressures are normalized by the inlet total pressure. The measured pressures were obtained from static pressure taps in the hub and shroud surfaces close to the vane fillet. The computed loadings show good agreement 
with measured values which exhibit smooth acceleration through the vane passage with no apparent flow separation. After the $80 \%$ meridional distance at the shroud, the measurement does not show as much acceleration as observed in the prediction. Figure 4 shows the spanwise distribution of circumferentially averaged flow angle and total pressure at the stator exit. The total pressure was measured at eight circumferential locations with nine different locations in the spanwise direction covering one complete stator passage. The flow angle was deduced by curve fitting the pressure from the probe at different yaw locations. Both the flow angle and total pressure profiles predicted by ADPAC appear to have typical features of the MFT as also shown by Kirtley et al. [5] and exhibit the characteristics of the flow into the rotor inlet. It is not clear how much the cutback of the rotor for the stator exit survey affected the discrepancy between predicted and measured values and a test for the stator alone is desirable to clarify this issue. It is also possible that the proximity of the survey probe to the rotor leading edge may influence the accuracy of the measurements.

Blade loadings and rotor exit flow characteristics are presented in Figures 5 and 6 . The blade surface static pressure distribution in Figure 5 shows computed values at three different spanwise locations. There are no data available for comparison. Note the substantial increase of the loading from hub to shroud beyond the $40 \%$ meridional chord. This is in contrast to the typical loading pattern for the radial turbine where most of the blade loading is imposed within the first half of the blade. The pressure mismatch around the leading edge at the $10 \%$ span indicates a negative incidence angle which may contribute to the performance loss of the rotor. The static pressure here becomes relatively flat after $20 \%$ meridional chord. Spanwise profiles of circumferentially averaged flow angle and total pressure at the rotor exit are depicted in Figure 6. The rotor exit survey was performed using the cobra probe in the null position at the plane of 0.33 inch downstream from the rotor trailing edge. Flow angles are defined as being positive when the tangential component of absolute velocity vector is in the direction of rotor rotation. Note the constant shifting of flow angles between data and ADPAC computation. Due to the proximity of the survey plane to the rotor trailing edge the flow is still very much nonuniform and this may result in the blockage effect in measurement. Predicted total pressure ratio by ADPAC show reasonable agreement with data and adequately catch the effect of the tip leakage flow. The big discrepancy between design intent and test data clearly indicates the deficiency of the design code which is based on radial turbine correlations.

The potential sources of the performance loss due to the leakage vortex and secondary 
flow motion may be qualitatively investigated by entropy contours as depicted in Figure 7. The entropy contours at various crossflow planes are shown at several meridional locations normalized by the chord length of the rotor blade. At 10 percent of meridional chord, high entropy generation due to the shroud leakage flow along the suction side is evident. At 50 percent of meridional chord, this tip leakage vortex is enlarged circumferentially and interacts with the low momentum fluid at hub which is drawn spanwise outward due to the pressure gradient across the span. The high entropy core due to the tip leakage vortex is evident at the 100 percent of meridional chord where the development of the wake also shows up. At the grid exit which is close to the rotor exit survey plane, the high entropy core near the shroud suction side still persists and contributes as a major source of performance loss. Observe the rapid mixing of the wake from the trailing edge to the rotor exit whereas the structure of the leakage vortex is preserved.

\section{Conclusions}

The aerodynamic performance of a high-work, mixed flow turbine stage was analyzed using a threedimensional Navier-stokes code, ADPAC. Predicted results of vane surface static pressures and spanwise distribution of flow angle and total pressure at stator and rotor exit agree favorably with measured data. The stator exit survey appears to be affected by the rotorstator interaction which has not been incorporated in the present study. Blade surface loadings indicate a negative incidence angle at the hub which may contribute to the discrepancy of the performance between measurement and design intent. Comparison of present analysis and design intent with data demonstrates that the design intent which is an axisymmetric code with radial turbine correlations does not properly capture the loss mechanism due to tip leakage vortex and secondary flow motion. The entropy contours predicted by present analysis clearly, if not quantitatively, indicate these important mechanisms of performance loss. Considering most of the industry design codes heavily rely on axisymmetric analysis with empirically-driven loss correlations, present study demonstrates that future direction in turbine design should embrace the capability of the three dimensional analysis code.

\section{Acknowledgments}

Authors express special thanks to Drs. L. Larosiliere and C. Miller for many helpful discussions. Sincere thanks are also given to Dr. L. Larosiliere for checking the manuscript.

\section{$\underline{\text { References }}$}

1. Rodgers, C., "Review of Mixed Flow and Radial Turbine Options," AIAA Paper No. 902414 
2. Okapuu, U., "Design and Aerodynamic Performance of $a$ Small Mixed-Flow Gas Generator Turbine," AGARD CP-421, May 1987, pp. 16-1-16-11.

3. Civinskas, K.C., "Aerodynamic Design Analysis of a Mixed-Flow, High specific Work Turbine," presented as AIAA oral Presentation No. 851275 at the 21st AIAA Joint Propulsion Conference, Monterey, CA, July 8-11, 1985.

4. Boyle, R.J., Haas, J.E., and Katsanis, T., "Comparison Between Measured Turbine stage Performance and the Predicted Performance Using Quasi-3D Flow and Boundary Layer Analyses," NASA TM-83640, 1984.

5. Kirtley, K.R., Beach, T.A., and Rogo, C., "Aeroloads and Secondary Flows in a Transonic Mixed-Flow Turbine Stage, "ASME Journal of Turbomachinery, Vol. 115, July 1993.

6. Adamczyk, J.J., "Model Equation for Simulating Flows in Multistage Turbomachinery," ASME Paper No. 85-GT-226, 1985. 7. Baldwin, B.S., and Lomax, H., "Thin Layer Approximation and Algebraic Model for Separated Turbulent Flows," AIAA Paper No. 78-257, Jan. 1978.

8. Hall, E.J., and Delaney, R.A., "Investigation of Advanced Counterrotation Blade Configuration Concepts for High Speed Turboprop systems, Task 5-Unsteady Counterrotation Ducted Propfan Analysis, Final Report," NASA CR-187126, Jan. 1993.

9. Chima, R.V., "Viscous ThreeDimensional Calculations of Transonic Fan Performance," AGARD CP-510, Feb. 1992, pp. 21-1-21-19.

10. Alliedsignal Engines, "High-Work Mixed-Flow Turbine
Technology, Final Report," July, 1993. 
Table 1. MFT Test Conditions.

Test 1:Stage Performance Test

Test 2:Stator Exit Survey

\begin{tabular}{||l|c|c||}
\hline \multicolumn{1}{|c|}{ Test Conditions } & Test 1 & Test 2 \\
\hline Inlet Temperature, degree R & 756.7 & 729.34 \\
\hline Inlet Pressure, psia & 61.54 & 61.00 \\
\hline Corrected Speed, rpm & 31,396 & 31,396 \\
\hline Corrected Flow, lom/sec & 0.860 & 0.866 \\
\hline Stator Pressure Ratio, T-s & 1.859 & 1.865 \\
\hline Stage Pressure Ratio, T-s & 4.190 & 4.394 \\
\hline
\end{tabular}

Table 2. Comparison of MFT Stage Performance at Design Point

\begin{tabular}{||l|c|c||}
\hline & mass flow $(1 \mathrm{bm} / \mathrm{sec})$ & stage T-T eff. \\
\hline Test & 0.860 & 0.888 \\
\hline Design Intent & 0.878 & 0.925 \\
\hline ADPAC & 0.889 & 0.898 \\
\hline
\end{tabular}




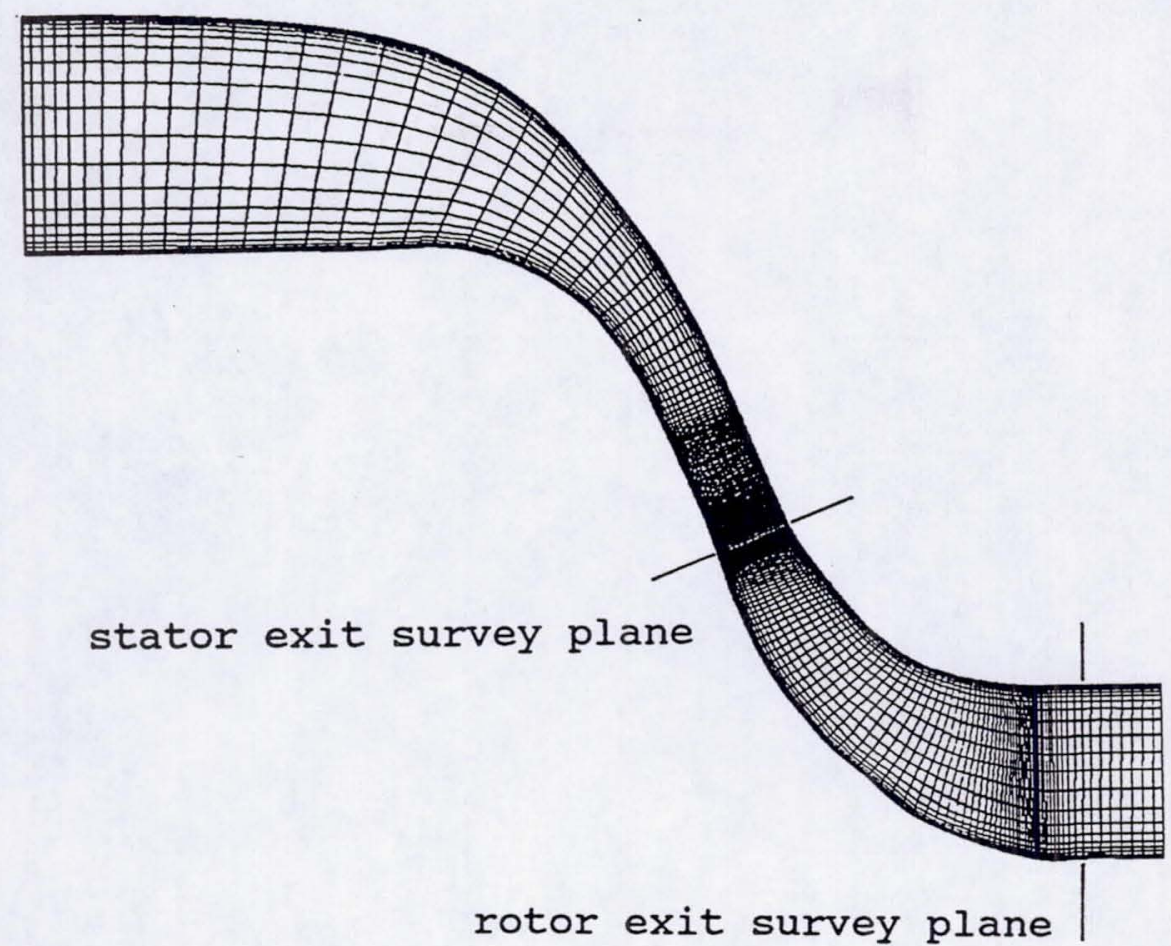

(a) Axisymmetric view of computational grid.

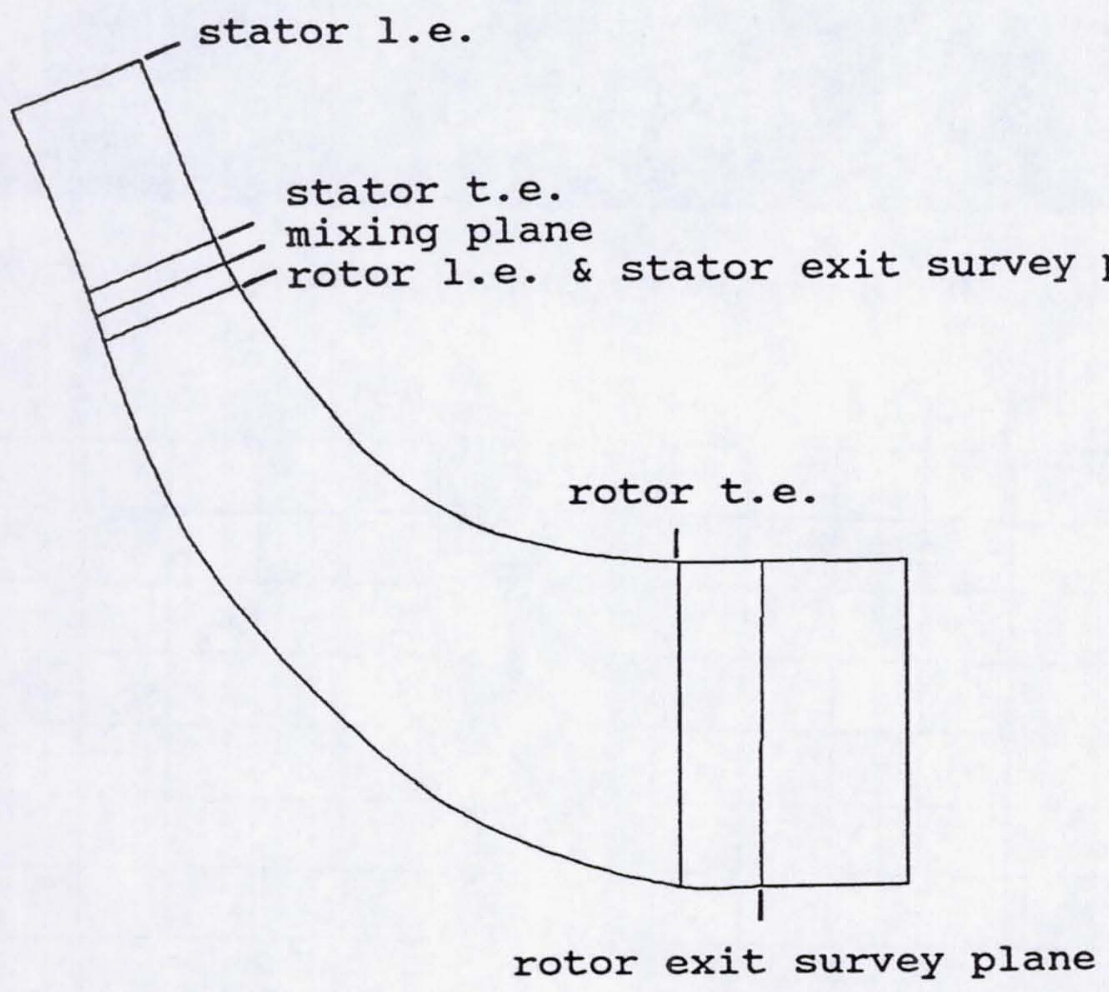

(b) stator and rotor geometry.

Figure 1. MFT geometry. 


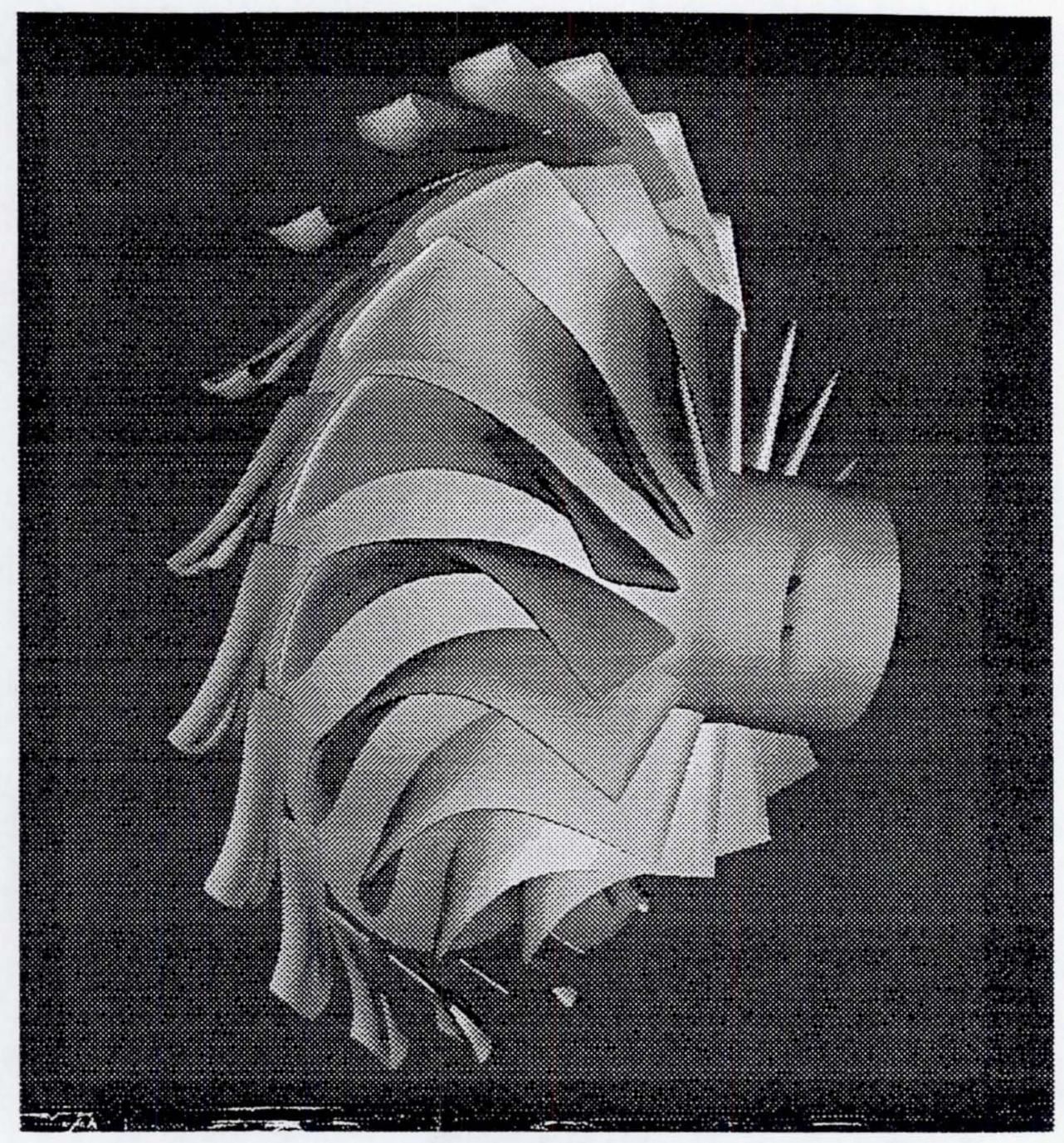

Figure 2. MFT geometry generated by computer graphics.

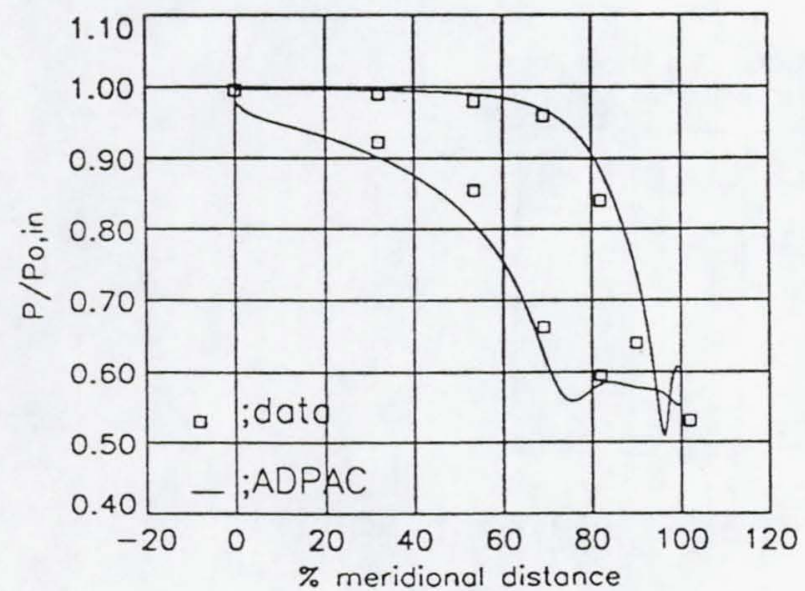

(a) Hub

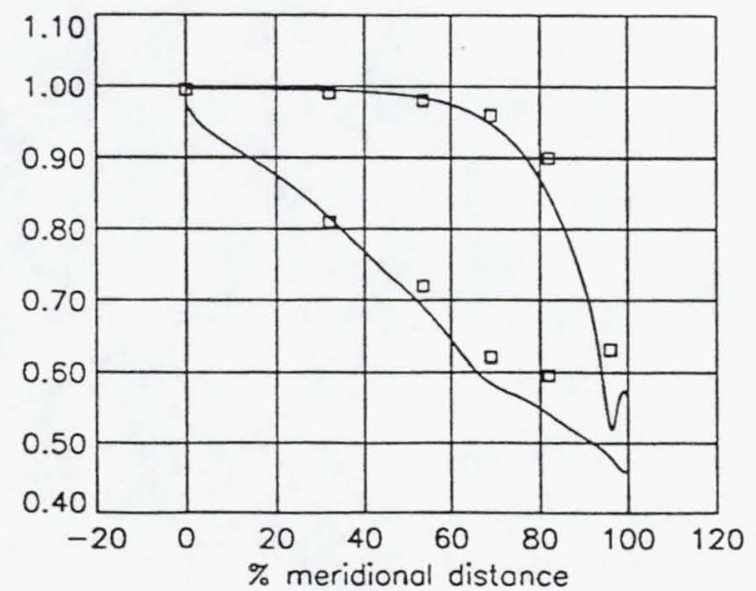

(b) Shroud

Figure 3. MFT vane surface static pressure distribution 


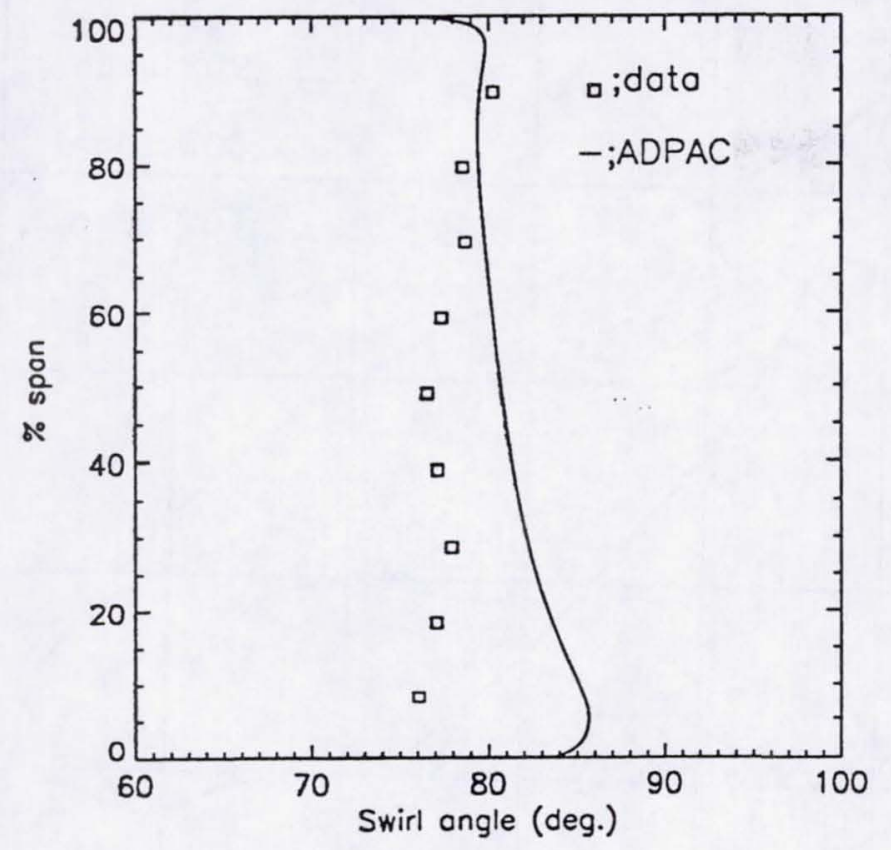

(a) Swirl angle

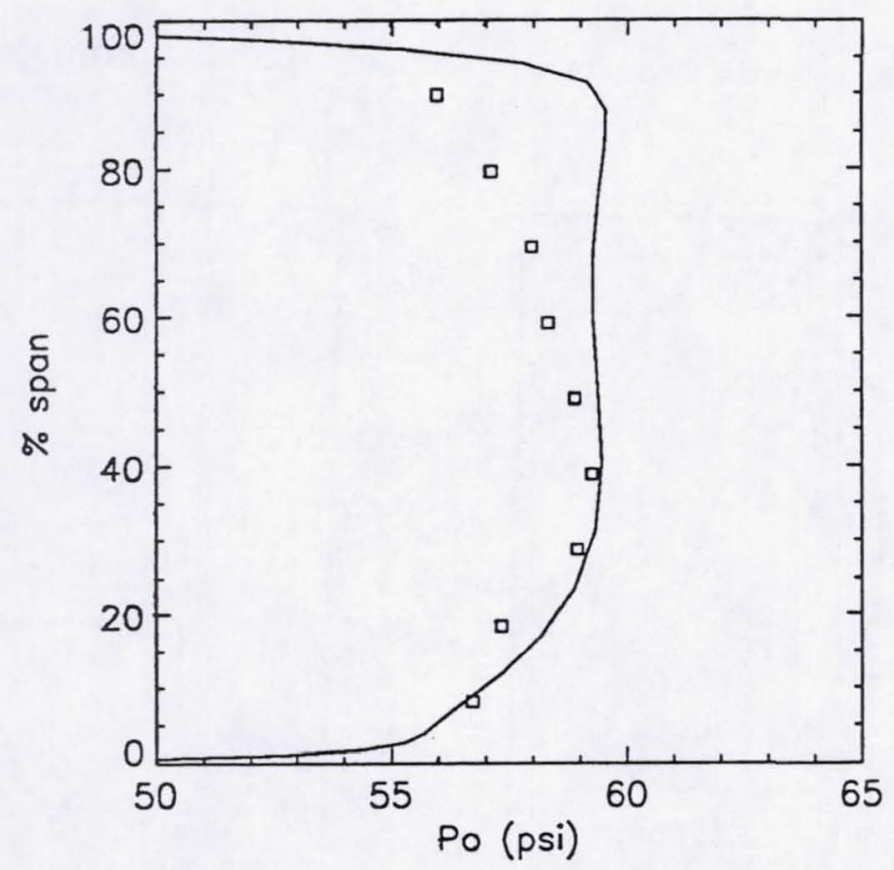

(b) Total pressure

Figure 4. Spanwise quantities at the stator exit. 


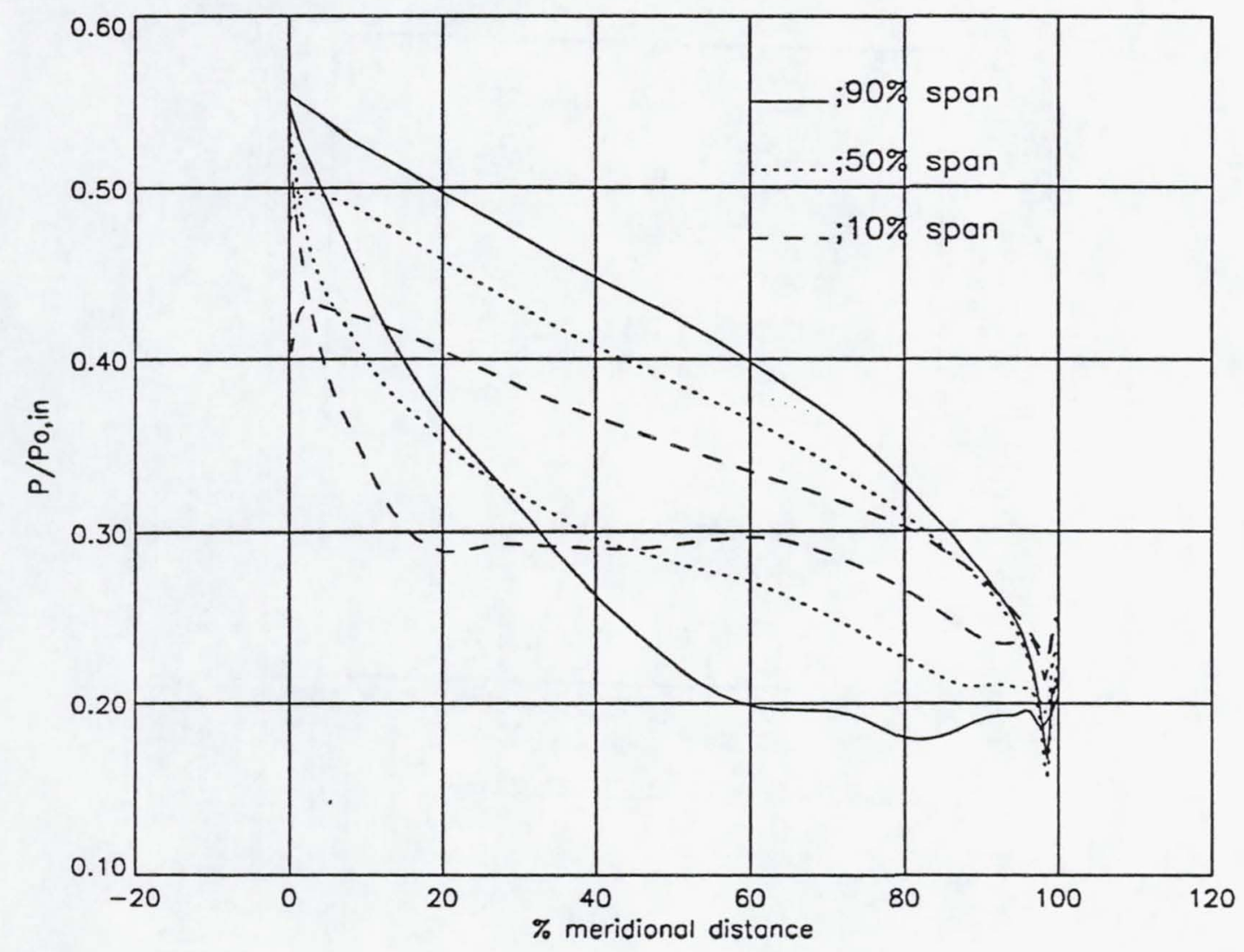

Figure 5. MFT blade loadings at various spanwise locations.

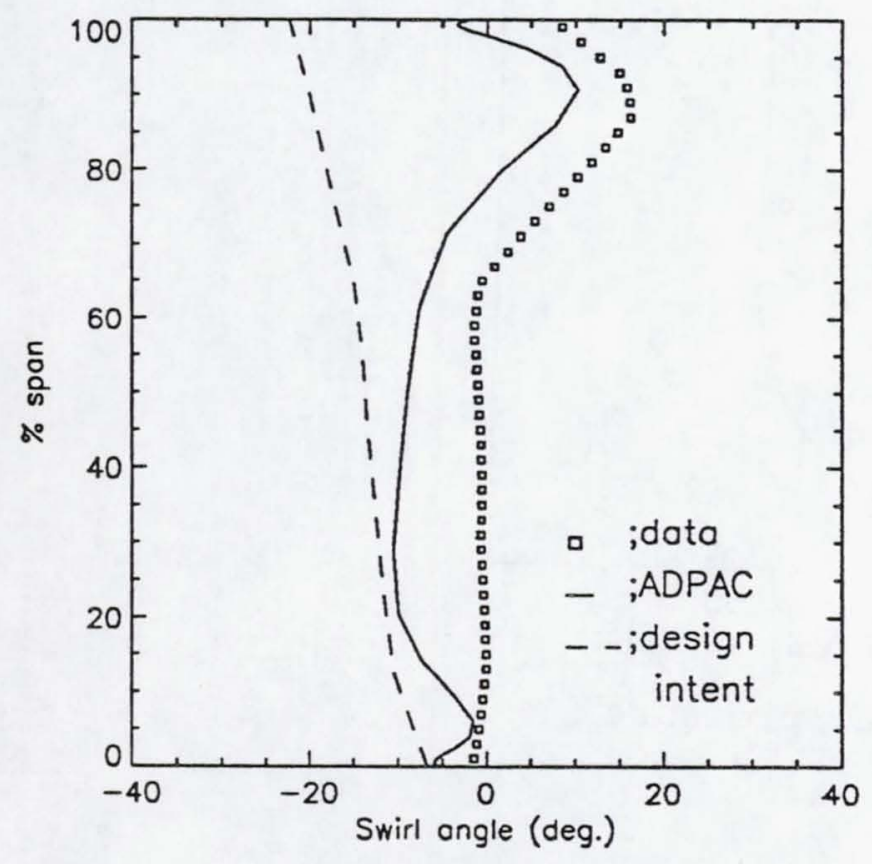

(a) Swirl angle

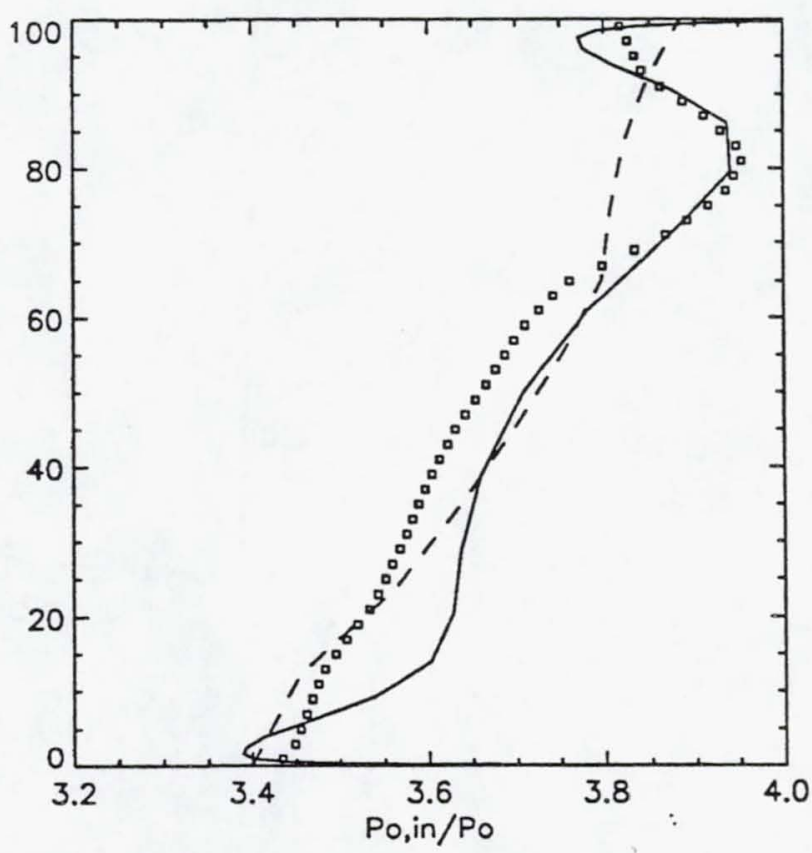

(b) Total pressure ratio

Figure 6. Spanwise quantities at the rotor exit. 


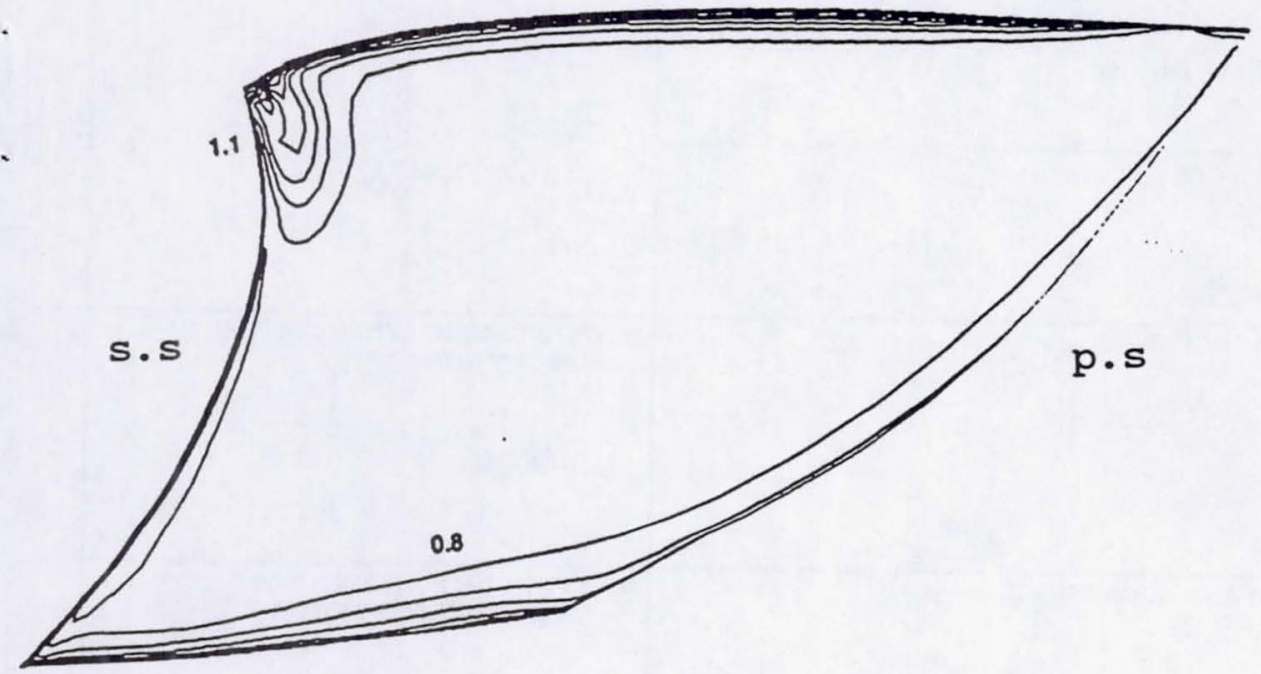

$10 \%$ meridional chord

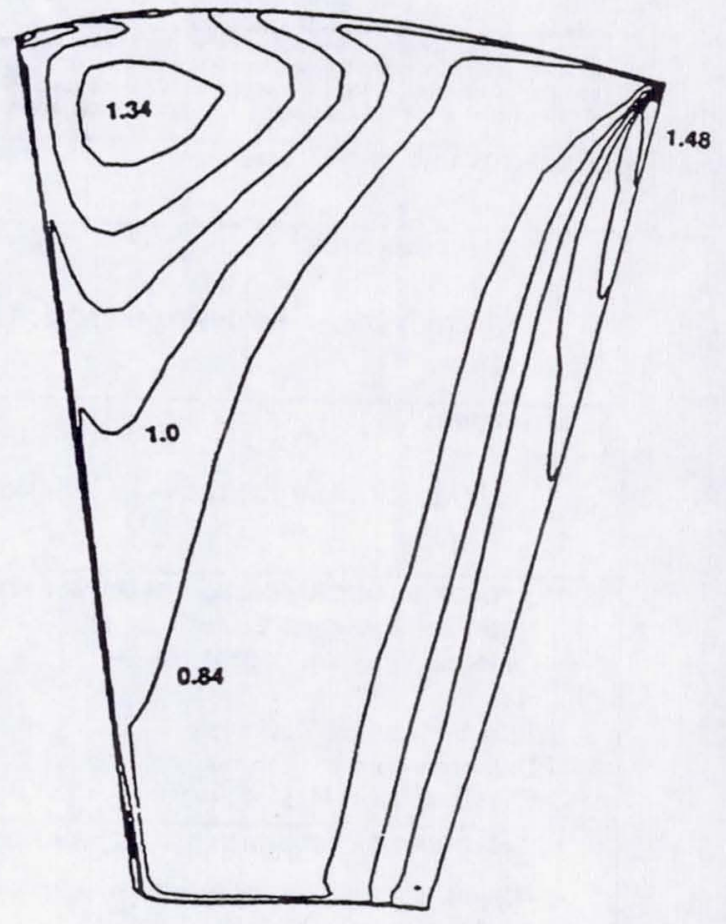

$100 \%$ meridional chord

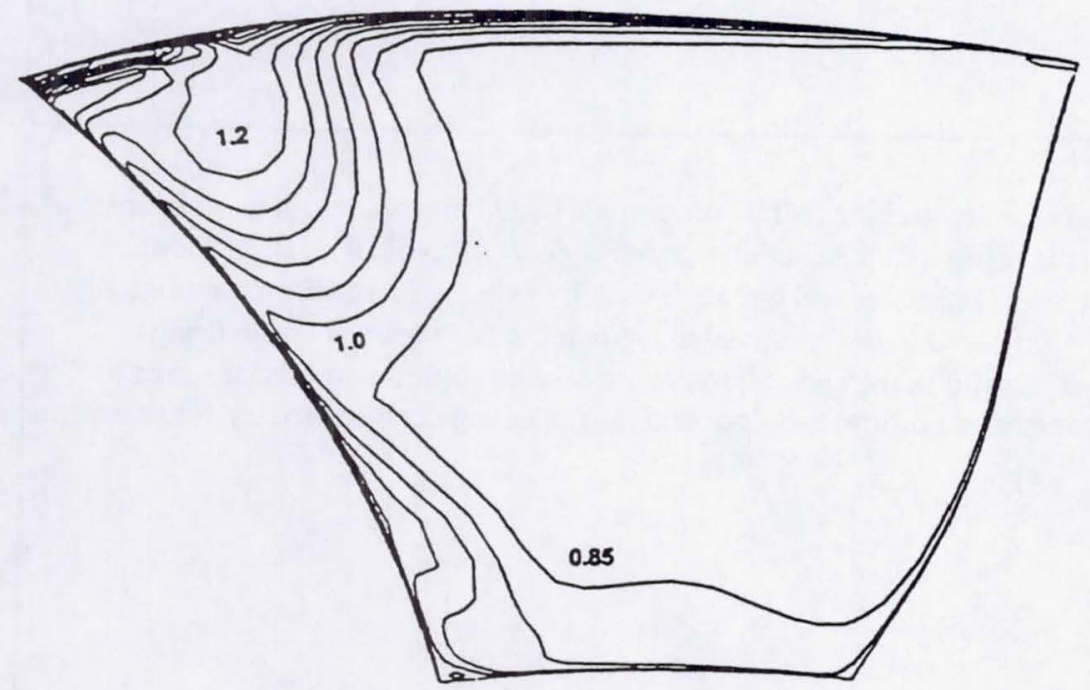

$50 \%$ meridional chord

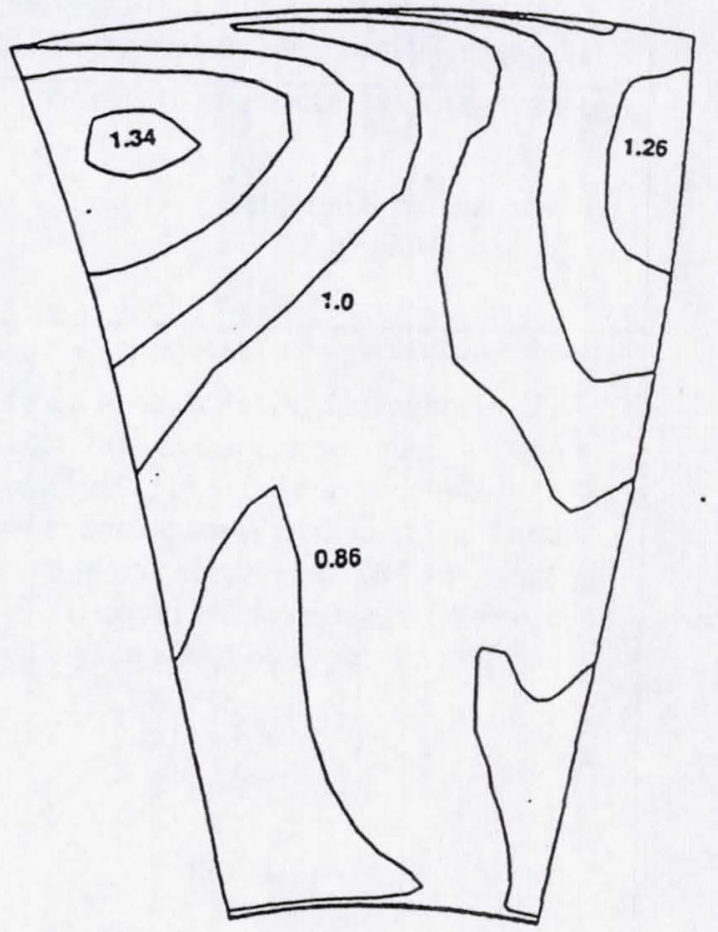

rotor exit

Figure 7. Entropy contours at various crossflow planes. 
Public reporting burden for this collection of information is estimated to average 1 hour per response, including the time for reviewing instructions, searching existing data sources, gathering and maintaining the data needed, and completing and reviewing the collection of information. Send comments regarding this burden estimate or any other aspect of this collection of information, including suggestions for reducing this burden, to Washington Headquarters Services, Directorate for Information Operations and Reports, 1215 Jefte

\begin{tabular}{|l|l|l|}
\hline 1. AGENCY USE ONLY (Leave blank) & $\begin{array}{c}\text { 2. REPORT DATE } \\
\text { July } 1994\end{array}$ & $\begin{array}{r}\text { 3. REPORT TYPE AND DATES COVERED } \\
\text { Technical Memorandum }\end{array}$ \\
\hline
\end{tabular}

4. TITLE AND SUBTITLE

5. FUNDING NUMBERS

An Aerodynamic Analysis of a Mixed Flow Turbine

6. AUTHOR(S)

WU-505-62-10

1L162211A47A

Chan M. Kim and Kestutis C. Civinskas

7. PERFORMING ORGANIZATION NAME(S) AND ADDRESS(ES)

NASA Lewis Research Center

8. PERFORMING ORGANIZATION

Cleveland, Ohio 44135-3191

and

Vehicle Propulsion Directorate REPORT NUMBER

US. Army Research Laboratory

E-9008

Cleveland, Ohio 44135-3191

9. SPONSORING/MONITORING AGENCY NAME(S) AND ADDRESS(ES)

National Aeronautics and Space Administration

Washington, D.C. 20546-0001

and

U.S. Army Research Laboratory

Adelphi, Maryland 20783-1145

ARL-TR-572

11. SUPPLEMENTARY NOTES

Prepared for the 30th Joint Propulsion Conference cosponsored by AIAA, ASME, SAE, and ASEE, Indianapolis, Indiana, June 27-29, 1994. Chan M. Kim, NASA Lewis Research Center, and Kestutis C. Civinskas, Vehicle Propulsion Directorate, U.S. Army Research Laboratory, NASA Lewis Research Center. Responsible person, Chan M. Kim, organization code 2760, (216) $433-8715$.

12a. DISTRIBUTION/AVAILABILITY STATEMENT

12b. DISTRIBUTION CODE

Unclassified - Unlimited

Subject Category 07

\section{ABSTRACT (Maximum 200 words)}

The aerodynamic performance of a high-work mixed flow turbine (MFT) is computed and compared with experimental data. A three dimensional (3-D) viscous analysis is applied to the single stage MFT geometry with a relatively long upstream transition duct. Predicted vane surface static pressures and circumferentially averaged spanwise quantities at stator and rotor exits agree favorably with data. Compared to the results of axisymmetric flow analysis from design intent, the 3-D computation agrees much better especially in the endwall regions where throughflow prediction fails to assess the loss mechanism properly. Potential sources of performance loss such as tip leakage and secondary flows are also properly captured by the analysis.

\begin{tabular}{|c|c|}
\hline 14. SUBJECT TERMS \\
Mixed flow turbine; Aerodynamic performance; Three-di \\
\hline $\begin{array}{c}\text { 17. SECURITY CLASSIFICATION } \\
\text { OF REPORT } \\
\text { Unclassified }\end{array}$ & $\begin{array}{c}\text { 18. SECURITY CLASSIFICATION } \\
\text { OF THIS PAGE } \\
\text { Unclassified }\end{array}$ \\
\hline
\end{tabular}

